- Supplementary File for -

\title{
The Role of Surface Sites on the Oscillatory Oxidation of Methanol on Stepped Pt[n(111) x (110)] Electrodes
}

V. Del Colle,1,2 P. B. Perroni, 1 J. M. Feliu,3 G. Tremiliosi-Filho,1 H. Varela1,4,**

I Institute of Chemistry of São Carlos, University of São Paulo POBox 780, 13560-970, São Carlos, SP, Brazil.

2 Universidade Federal de Alagoas - Campus Arapiraca, Av. Manoel Severino Barbosa 57309-005, Arapiraca, AL, Brazil.

3 Instituto de Electroquímica, Universidad de Alicante, Apdo. 99, E-03080 Alicante, Spain. 4 Max-Planck Institute for the Physics of Complex Systems, Nöthnitzer Str. 38 01187 Dresden, Germany.

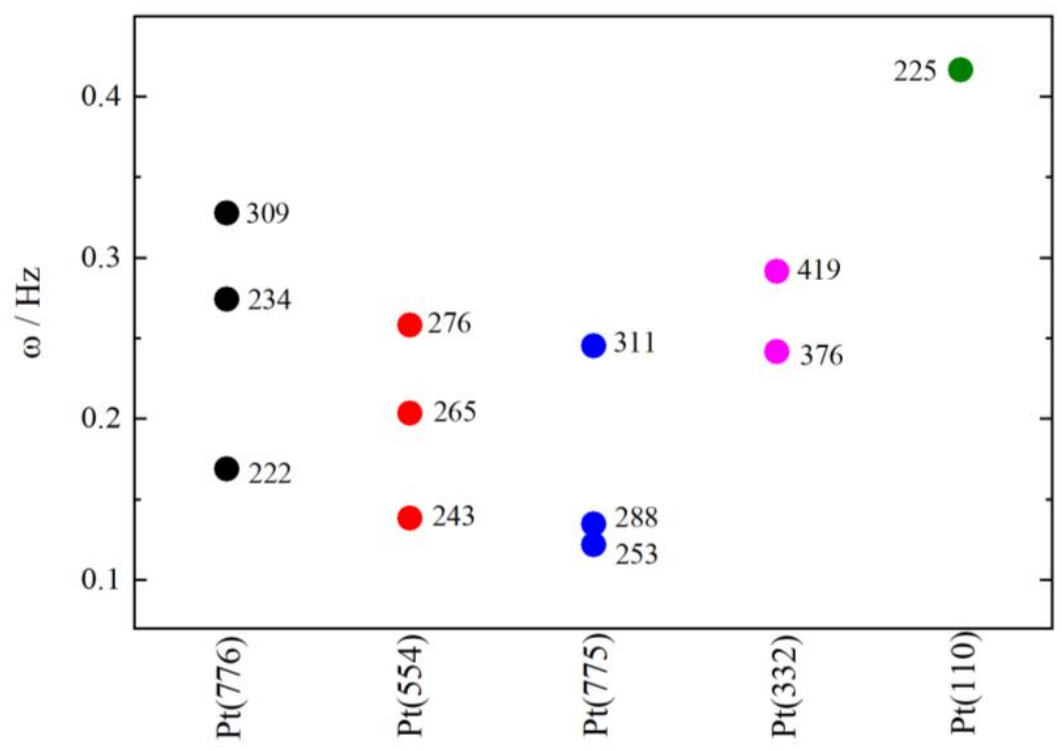

Figure S1: Frequencies of quasi-harmonic oscillations near the bifurcation point for different platinum surfaces and for distinct applied currents (values inside the figure in $\mu \mathrm{Acm}-2$ ). Electrolyte: aqueous solution containing $\left[\mathrm{H}_{3} \mathrm{COH}\right]=0.20 \mathrm{~mol} \mathrm{~L}-1$ and $\left[\mathrm{H}_{2} \mathrm{SO}_{4}\right]=0.5 \mathrm{~mol} \mathrm{~L}-1$. 


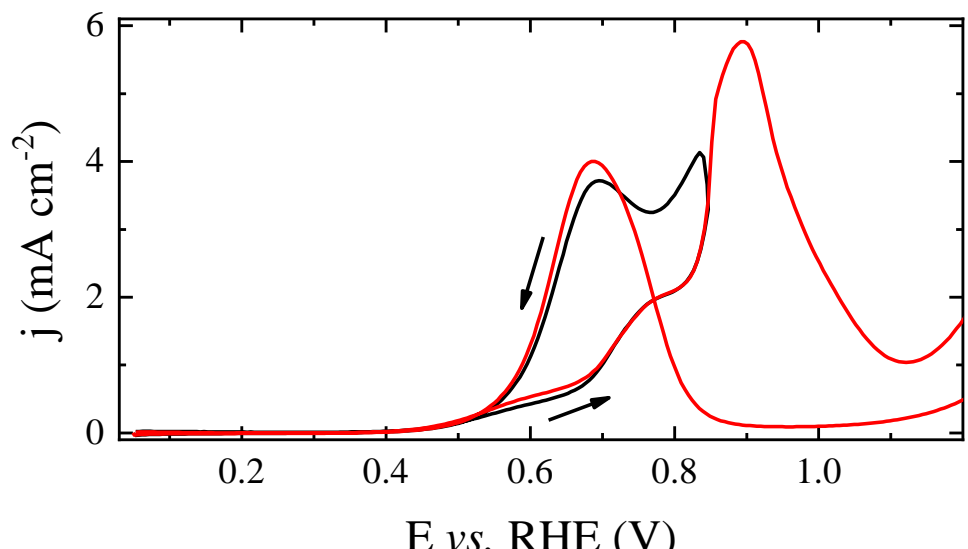

Figure S2: Voltammetric profiles of the electro-oxidation of methanol on polycrystalline platinum at 50 (red) and $20 \mathrm{mVs}-1$ (black). Electrolyte: aqueous solution containing $\left[\mathrm{H}_{3} \mathrm{COH}\right]=10 \mathrm{~mol} \mathrm{~L}-1$ and $\left[\mathrm{H}_{2} \mathrm{SO}_{4}\right]=0.5$ mol L-1. 


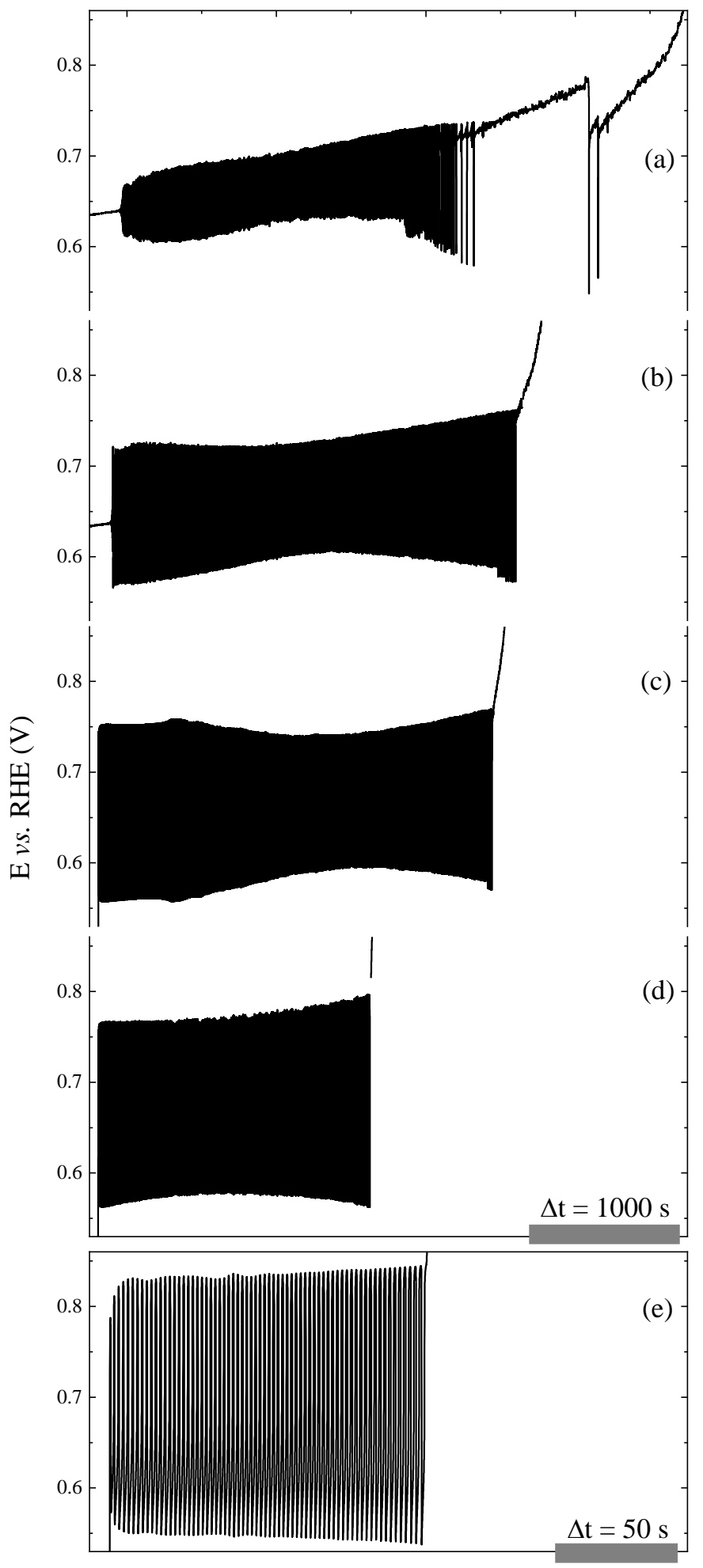

Figure S3: Galvanostatic electro-oxidation of methanol on $\operatorname{Pt}(776)$ at (a) 0.492, (b) 0.615, (c) 0.738, (d) 0.985, and (e) $1.60 \mathrm{mAcm}-2$. Electrolyte: aqueous solution containing $\left[\mathrm{H}_{3} \mathrm{COH}\right]=10 \mathrm{~mol} \mathrm{~L}-1$ and $\left[\mathrm{H}_{2} \mathrm{SO}_{4}\right]=0.5$ mol L-1. 

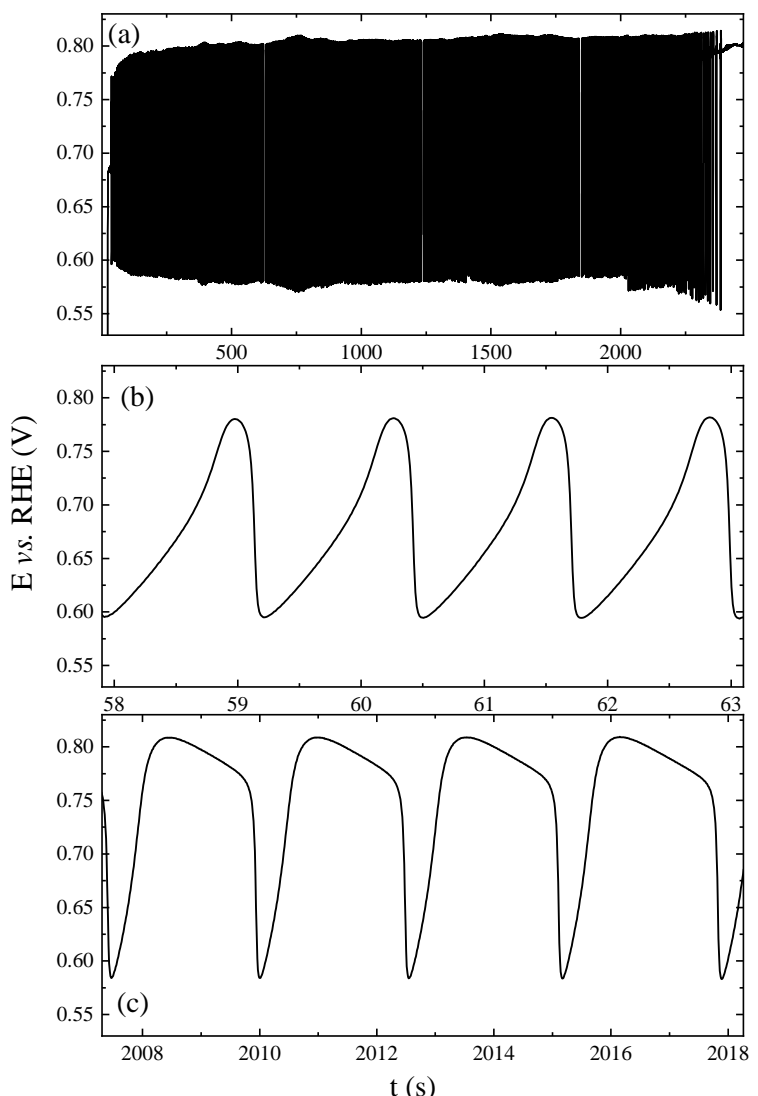

Figure S4: Potential time-traces of the galvanostatic electro-oxidation of methanol on polycrystalline platinum at $1.445 \mathrm{mAcm}-2$. Electrolyte: aqueous solution containing $\left[\mathrm{H}_{3} \mathrm{COH}\right]=10 \mathrm{~mol} \mathrm{~L}-1$ and $\left[\mathrm{H}_{2} \mathrm{SO}_{4}\right]=0.5 \mathrm{~mol} \mathrm{~L}-1$. 

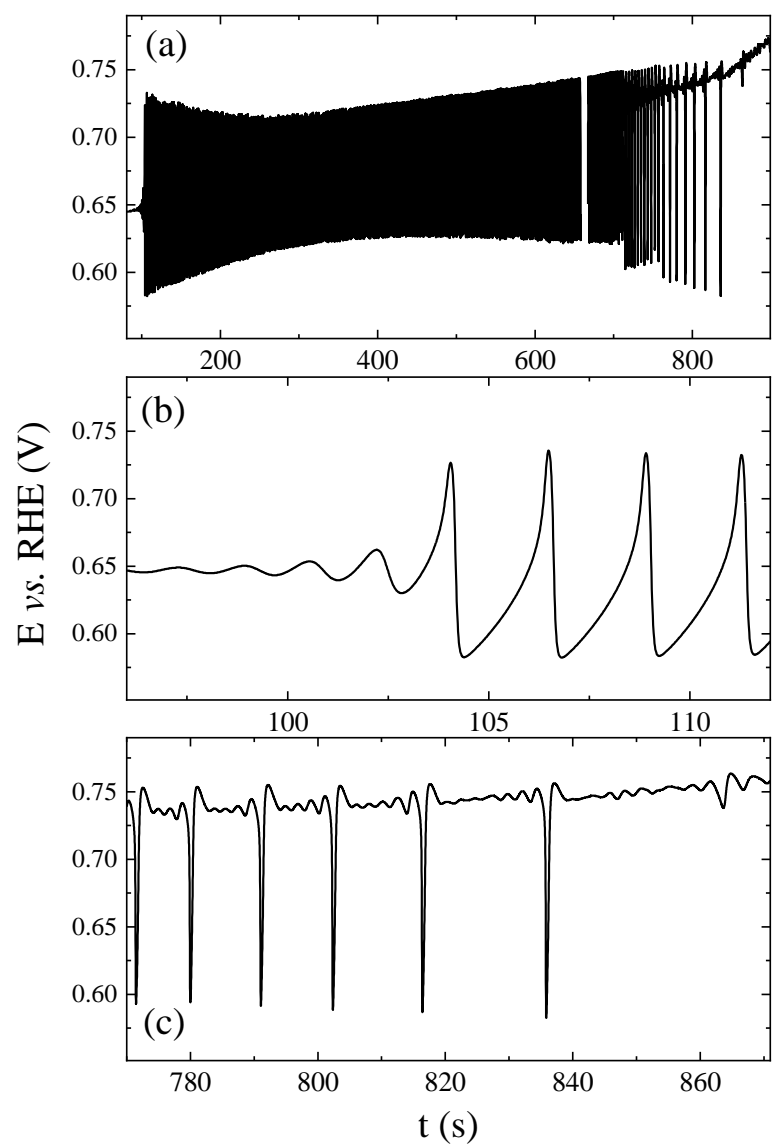

Figure S5: Oscillations in the electro-oxidation of methanol on a $\operatorname{Pt}(554)$ electrode, at $0.608 \mathrm{mAcm}-2$. Electrolyte: aqueous solution containing $\left[\mathrm{H}_{3} \mathrm{COH}\right]=10 \mathrm{~mol} \mathrm{~L}-1$ and $\left[\mathrm{H}_{2} \mathrm{SO}_{4}\right]=0.5 \mathrm{~mol} \mathrm{~L}-1$. 


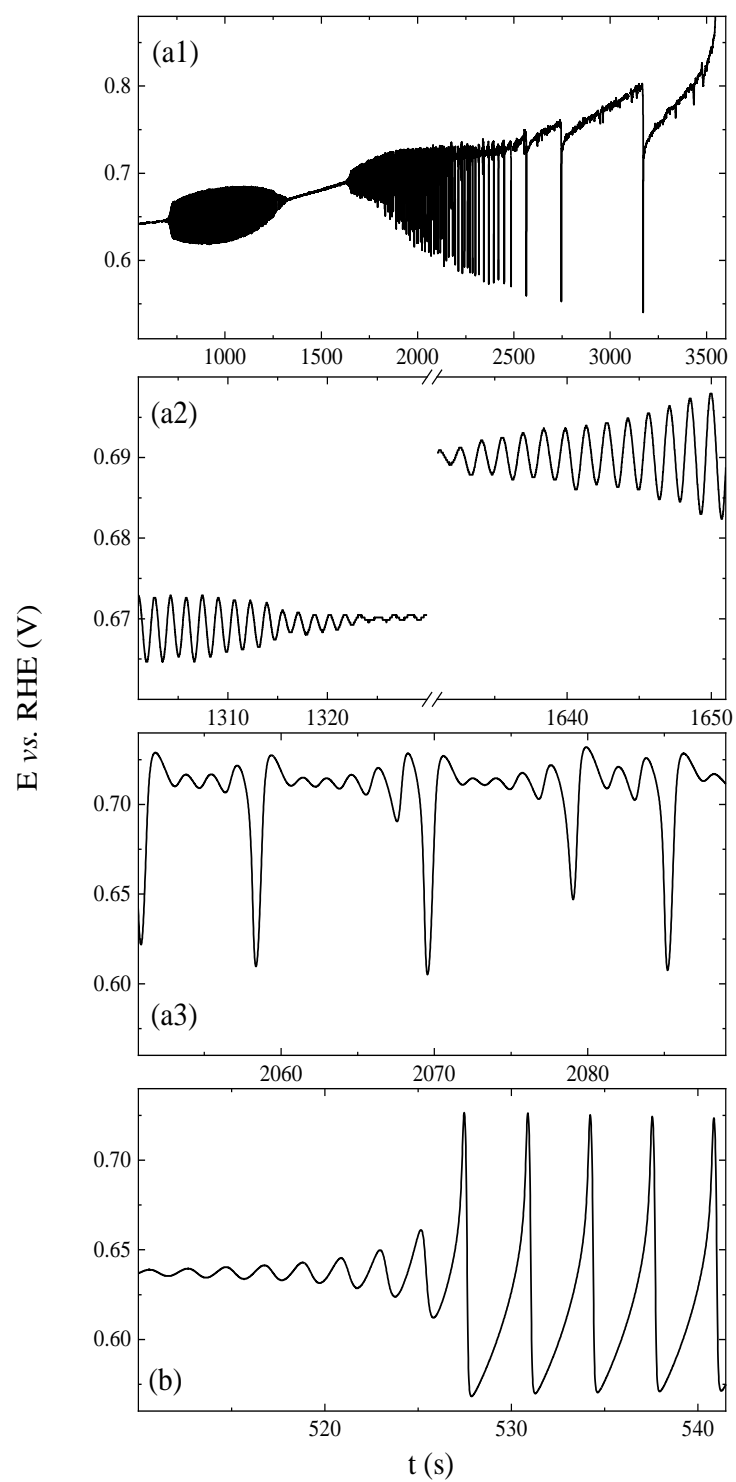

Figure S6: Oscillatory electro-oxidation of methanol on $\mathrm{Pt}(775)$ surface, at different applied currents: (a1) - (a3) $0.518 \mathrm{mAcm}-2$, and (b) $0.633 \mathrm{mAcm}-2$. Electrolyte: aqueous solution containing $\left[\mathrm{H}_{3} \mathrm{COH}\right]=10 \mathrm{~mol} \mathrm{~L}-1$ and $\left[\mathrm{H}_{2} \mathrm{SO}_{4}\right]=0.5 \mathrm{~mol} \mathrm{~L}-1$. 


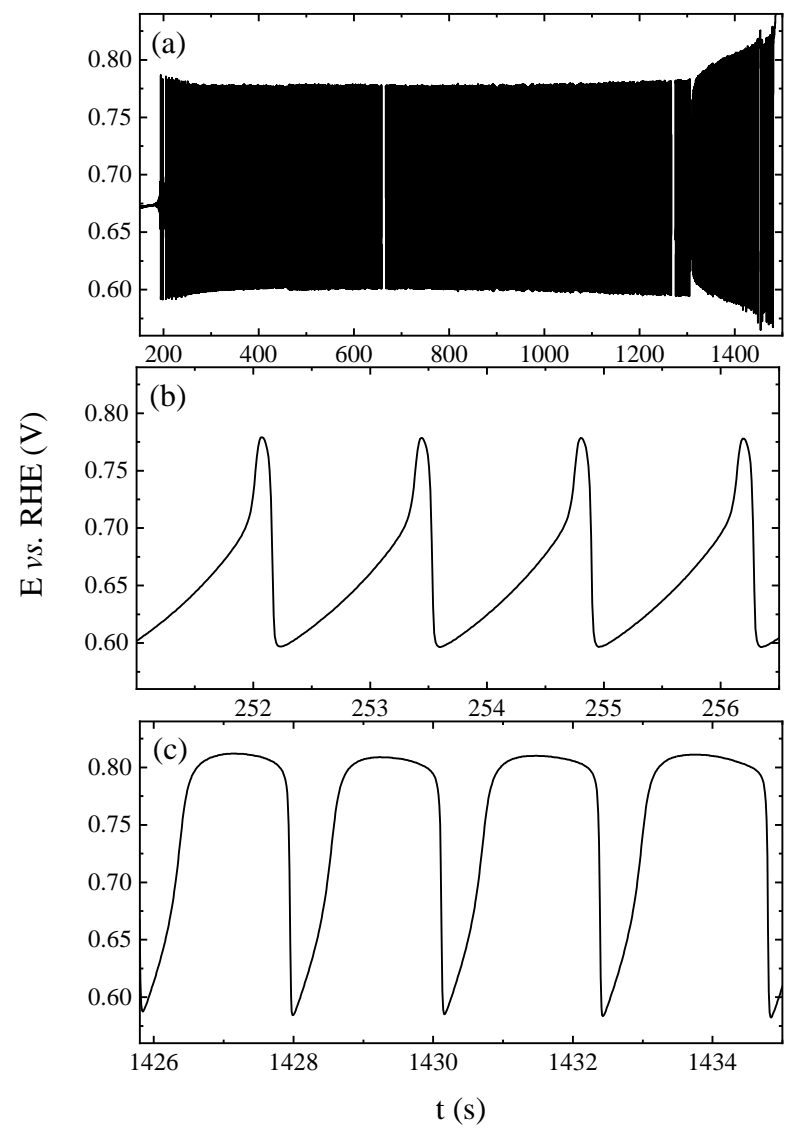

Figure S7: Oscillations in the electro-oxidation of methanol on a $\mathrm{Pt}(332)$ electrode, at $1.735 \mathrm{mAcm}-2$. Electrolyte: aqueous solution containing $\left[\mathrm{H}_{3} \mathrm{COH}\right]=10 \mathrm{~mol} \mathrm{~L}-1$ and $\left[\mathrm{H}_{2} \mathrm{SO}_{4}\right]=0.5 \mathrm{~mol} \mathrm{~L}-1$. 